\title{
"Use of Biological Solutions for Annular Healing: Dervan Platelet Fibrin Plug in Transforaminal Disc Surgery."
}

\author{
Dr.Sunil Nadkarni, Dr. Pavankumar Kohli, Dr. Satishchandra Gore, \\ Dr. Bhupesh Patel
}

\section{Introduction}

Biological treatment approaches recently have shown encouraging results in degenerative disc disease and additional relief in back and leg pain ${ }^{1}$.Stitchless endoscopic transforaminal surgery under local anaesthesia is a paradigm shift IN spine surgery for disc degeneration. ${ }^{2}$ Ability of a surgeon to identify pain generators in patient under local anaesthesiais its core. The "in vivo visualization" of etiopathology in degenerative lumbar spine and probing pain generators in real time revolutionized disc and spine surgery by increasing certainty of diagnosis and resulting treatment planning. ${ }^{3}$

One of the main limitation in transforaminal endoscopy is entry thru the annulus in foramen by blunt dilatation of the annular fibres creating a new rent in annulus. Comparison of using working cannula in annulus, a circular incision or a square incision in annulus has showed less destruction of annulus fibrosus by working cannula. Use of working cannula helps in maintenance of biomechanical strength and repair of annulus fibrosus. ${ }^{4}$ But a small residual annular defect needs treatment to close it at end of surgery.

Annular tear is a fundamental change in symptomatic disc to bring nuclear tissue in touch with neuro vascular supply of the disc. ${ }^{5}$ Chronic annular tear causes symptoms of back and leg pain. The main component is "inflammation". This has been variously treated by instilling locally active anti-inflammatory agent or removing cause of the inflammation, that is a trapped piece of nucleus in the torn annulus. The torn annulus needs treatment in form of a healing stimulus to relieve symptoms.

The present study is for both these issues. We utilised basic access of transforaminal endoscopy to access and visualize the pain generators and deliver anti-inflammatory "biological solution" at site of pain generation and second at end of intervention tried to cover the iatrogenic annular defect with a biological autologous solution.

IN our study standardised protocol and equipment to obtain a reasonable quality platelet fibrin plug was evolved. ${ }^{6,7,8,9,10}$ We have investigated if this autologous fibrin plug in and around annular tear and around the root can reduce early postoperative discomfort, pain and spasm and later help local healing of annular tear and rent and patient's symptomatic recovery.

\section{Method}

26 stitchless disc surgeries were done under local anaesthesia by inside out YEUNG and GORE technique ${ }^{2}$ for the standard indications under $\mathrm{C}$ arm guidance with the patient in prone position. The needle was introduced into the foraminal portion of the targeted disc from about $10-12 \mathrm{~cm}$ from the midline. Thereafter the insertion of guide wire then a dilator to bluntly dilate annular fibres to gain an entry inside the disc and finally a working cannula of $7.6 \mathrm{~mm}$ diameter was followed. A working channel endoscope [Gore system ${ }^{\circledR}$ Karl Storz endoscopy] was then introduced and targeted fragmentectomy was performed. ${ }^{11}$

\section{MRI of a Lumbar Herniated Dise}

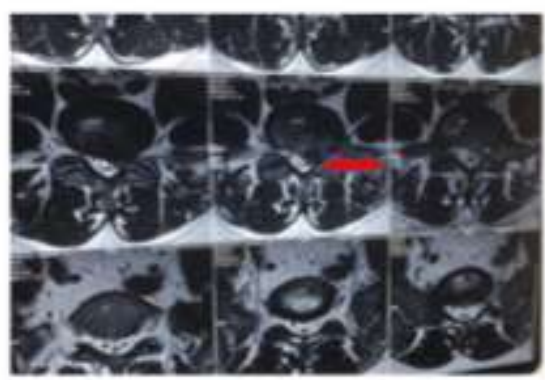

Avial Vlew: Hermiation Otserved at Level L.4-1.5
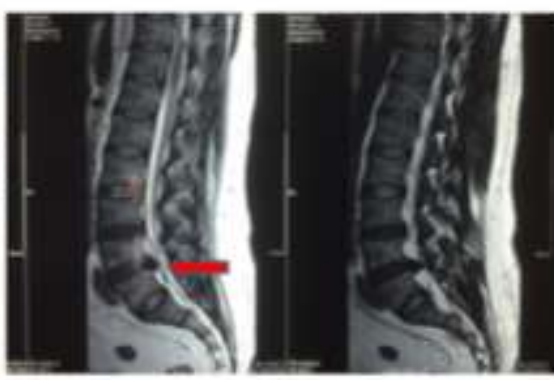

Sagittal Vhew: Hermiation Observed at Level L.4.LS 
Removed Intervertchal Disc Tisene
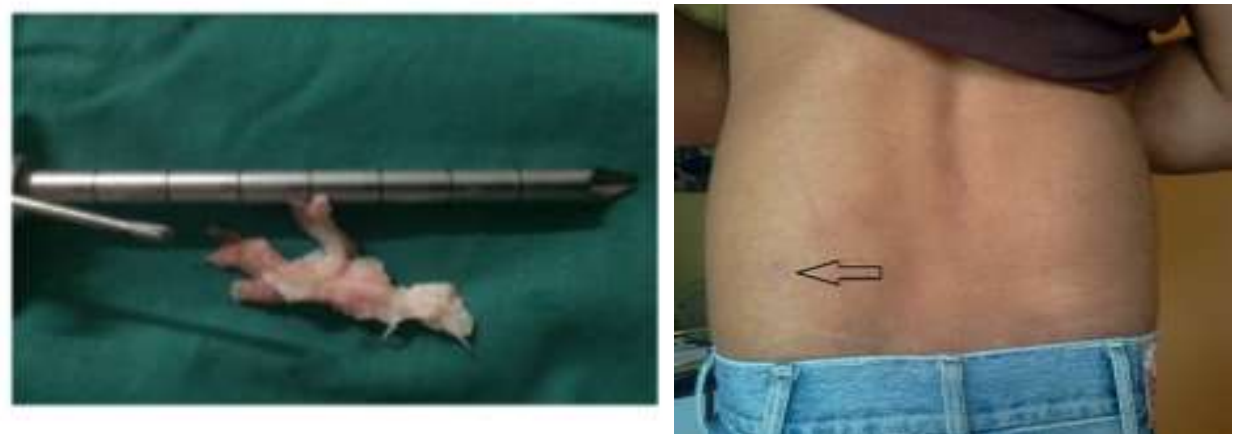

Pre-Operative MRI Depicts Problem We Treat by Stitchless Surgery Under Local Anaesthesia.Stitchless Surgery Scar at End of 1 Year. Left Side Access to Intervertebral Foramen atL45. Black Arrow

\section{Autologous platelet fibrin plugpreparation protocol in the present study.}

About $15 \mathrm{ml}$ of blood is drawn and immediately (before clotting cascade is triggered i.e. less than a minute and without any anticoagulant) transferred to a autoclaved plain glass tube with a cap and centrifuged at $3000 \mathrm{rpm}$ for 10 minutes. Thereafter a standing time in the centrifuge of 10 minutes is allowed. Using asepticprecautions, the tube is then uncapped by the circulating theatre personnel and the plug is lifted from the tube and is ready for insertion(Photograph 1,2) through working cannula at symptomatic annular tear or annular rent.This plug is placed in the cannula and pushed into the disc using the dilator (photograph 3,4,5)to seat it at annular defect or rent at the end of the procedure. Early outcome of surgery and use of plug was measured by VAS scale, Oswestry disability index.Case records were reviewed for any adverse events.

Photograph:1

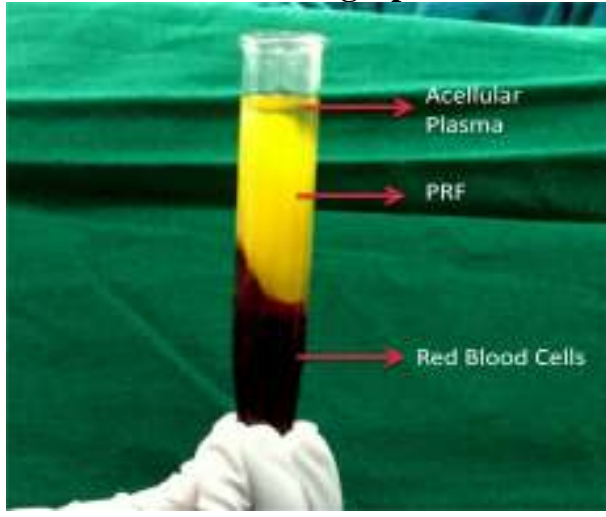

Photograph:2

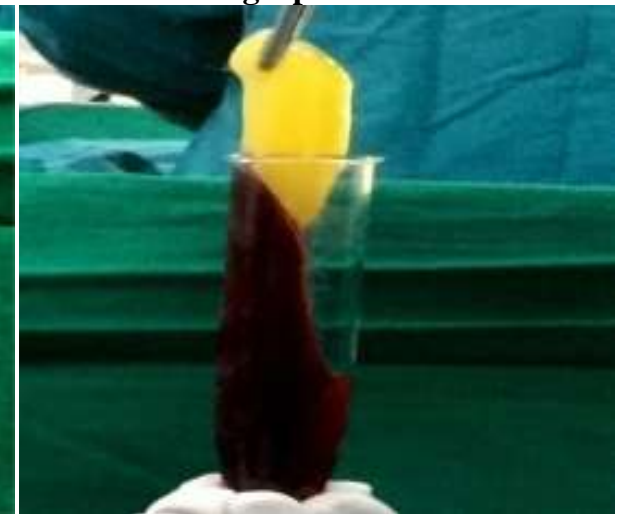

Photograph: 1.Blood in the tube after centrifugation of $3000 \mathrm{rpm}$ for $10 \mathrm{~min}$ and10min of standing time, separated in three fractions, upper fraction of acellular layer, middle of PRF and bottom fraction of RBC's 2.Platelet Rich Fibrin Plug immediately after standing time of 10min, ready for insertion.
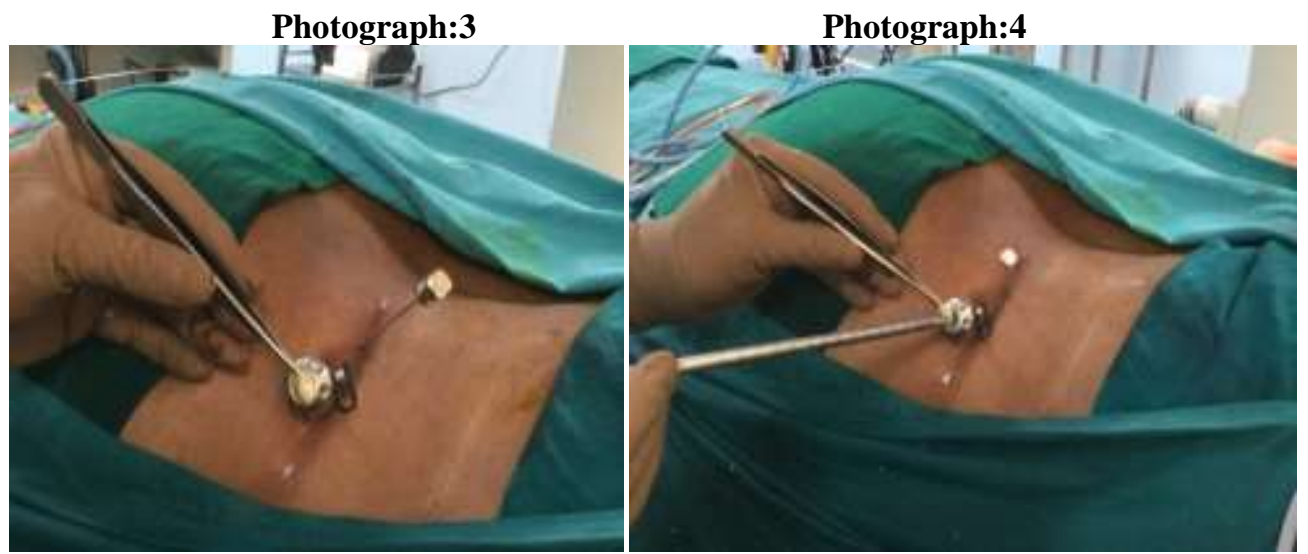


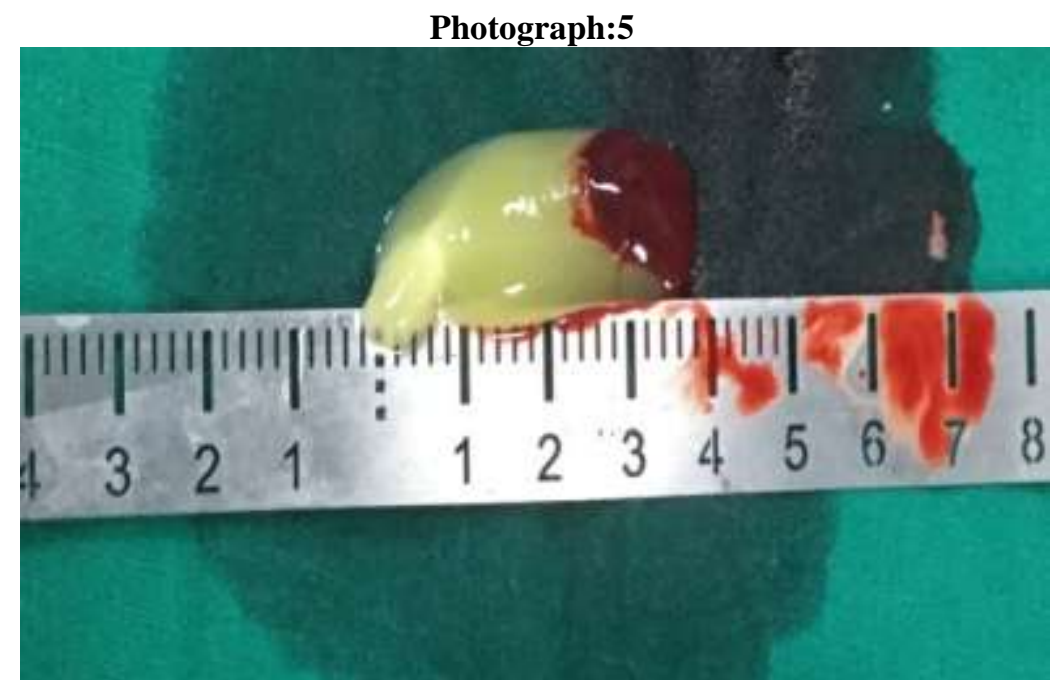

Photograph: 3. Platelet Rich Fibrin (PRF) plug inserted in the cannula at the end of stitchless disc surgery under local anaesthesia 4. Platelet Rich Fibrin (PRF) plug inserted into the disc through the cannula with the help of dilator pusher $5.3 .3 \mathrm{~cm}$ PRF plug obtained from $15 \mathrm{ml}$ whole blood volume, were directly inserted into the disc at site of annular tear and rent.

\section{Results}

Total 26 patient's surgical outcomes are presented in the study. $46 \%$ of the patients were males whereas $54 \%$ were the females with the average age of 38.7 years \& 58.7 years respectively. There were no adverse events in this study from the use of the platelet fibrin plug, hereafter called Dervan plug.

The mean VAS score for pain, showed significant improvement by $73 \%$ (Figure:1).

Fig: 1 VAS Score

Preoperative 9.3 and Postoperative 2

Modified version of ODI questionnaire was converted to local Marathi language for better understanding of local patients. The average pre-operative ODI was calculated as $83 \%$ whereas post-operative ODI score showed $62 \%$ decline to $21 \%$ score(Figure:2).

Fig:2 ODI score

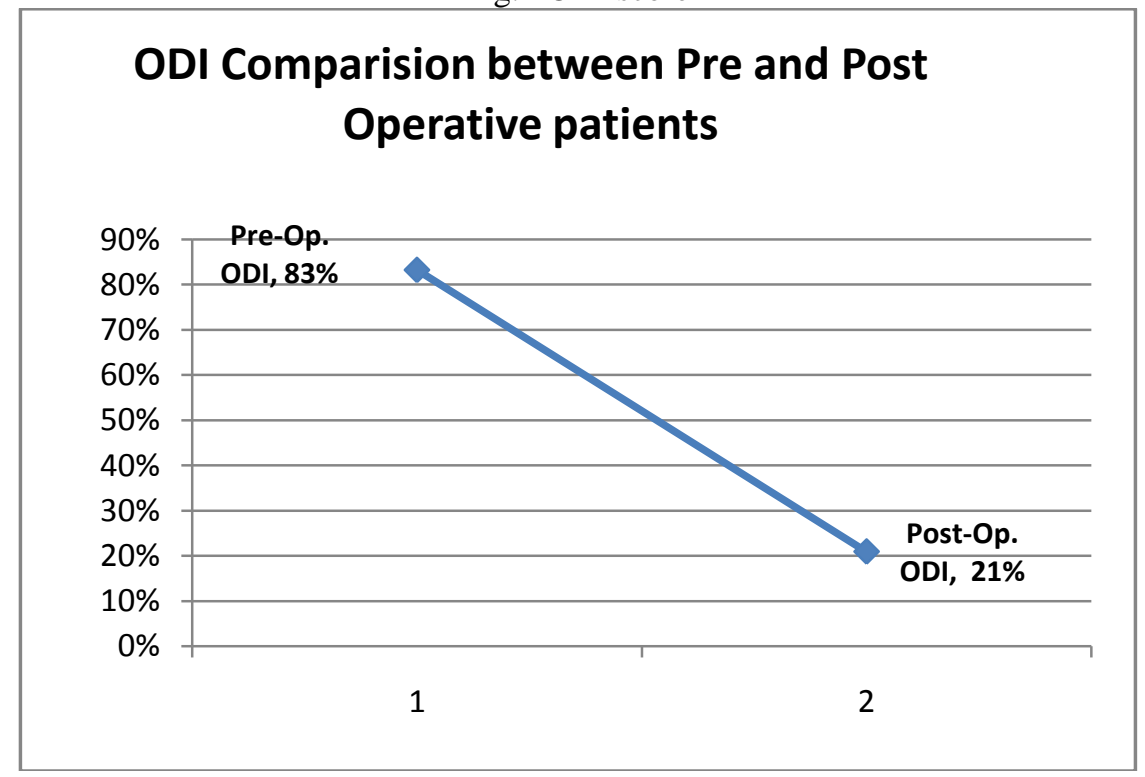

Figure: 2. ODI comparison between Pre and Post-Operative Average score. Graph shows $62 \%$ of average reduction in post-operative ODI Score, 


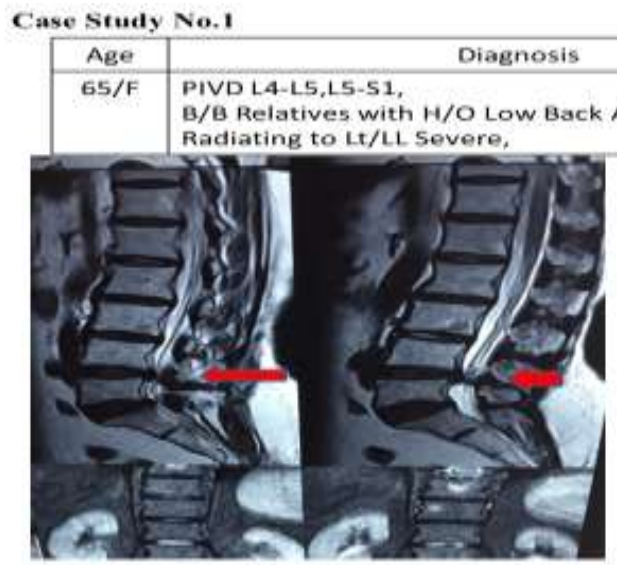

Pre Opt MR!
Procedure Done Percutaneous Lumbar Endoscopic Discectomy

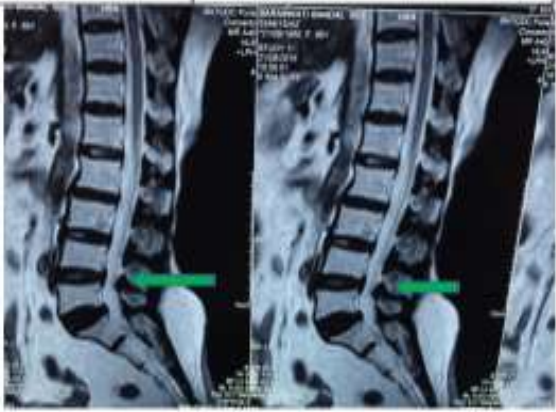

Post Opt MRI

Case Study No.2

\begin{tabular}{|c|l|c|}
\hline Age & \multicolumn{1}{|c|}{ Diagnosis } & Procedure Done \\
\hline 58/M & $\begin{array}{l}\text { PID L3-L4, L4-L5 } \\
\text { B/B Relatives with C/O: Low Back Ache Radiating to } \\
\text { Lt/L/L }\end{array}$ & $\begin{array}{l}\text { Lumbar Endoscopic } \\
\text { Discectomy }\end{array}$ \\
\hline
\end{tabular}
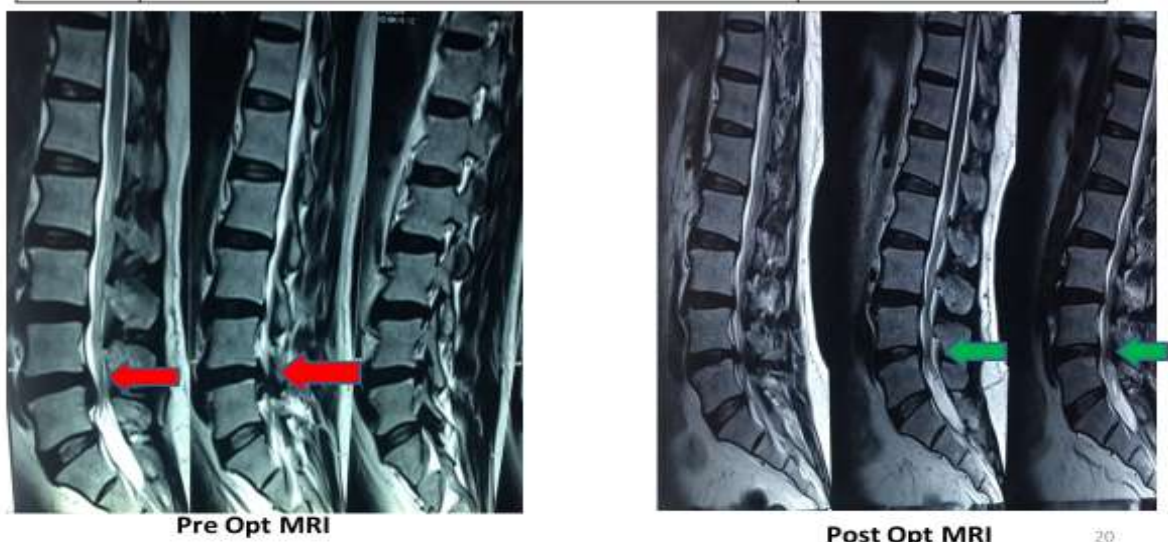

Post Opt MRI

Case Study No.3

\begin{tabular}{|c|l|l|}
\hline Age & \multicolumn{1}{|c|}{ Diagnosis } & \multicolumn{1}{|c|}{ Procedure Done } \\
\hline $53 / F$ & $\begin{array}{l}\text { L4-L5 PID } \\
\text { B/B Relatives with C/O: Low Back Ache Radiating to } \\
\text { Lt/L/L associated with weakness and T/N }\end{array}$ & $\begin{array}{l}\text { Percutaneous Lumbar } \\
\text { Endoscopic Discectomy }\end{array}$ \\
\hline
\end{tabular}
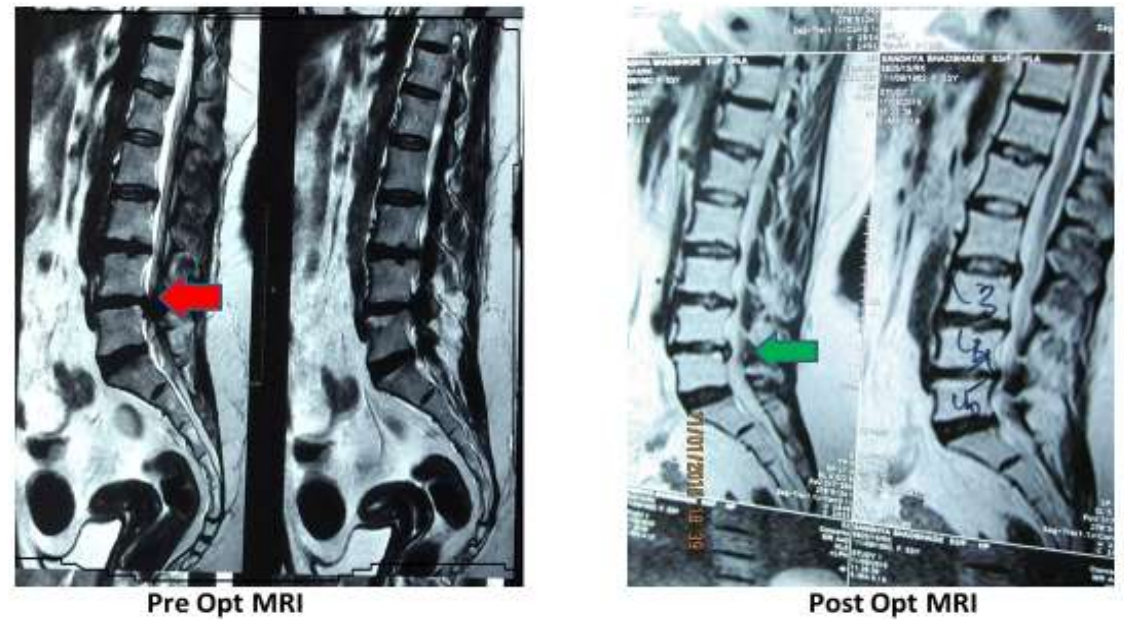


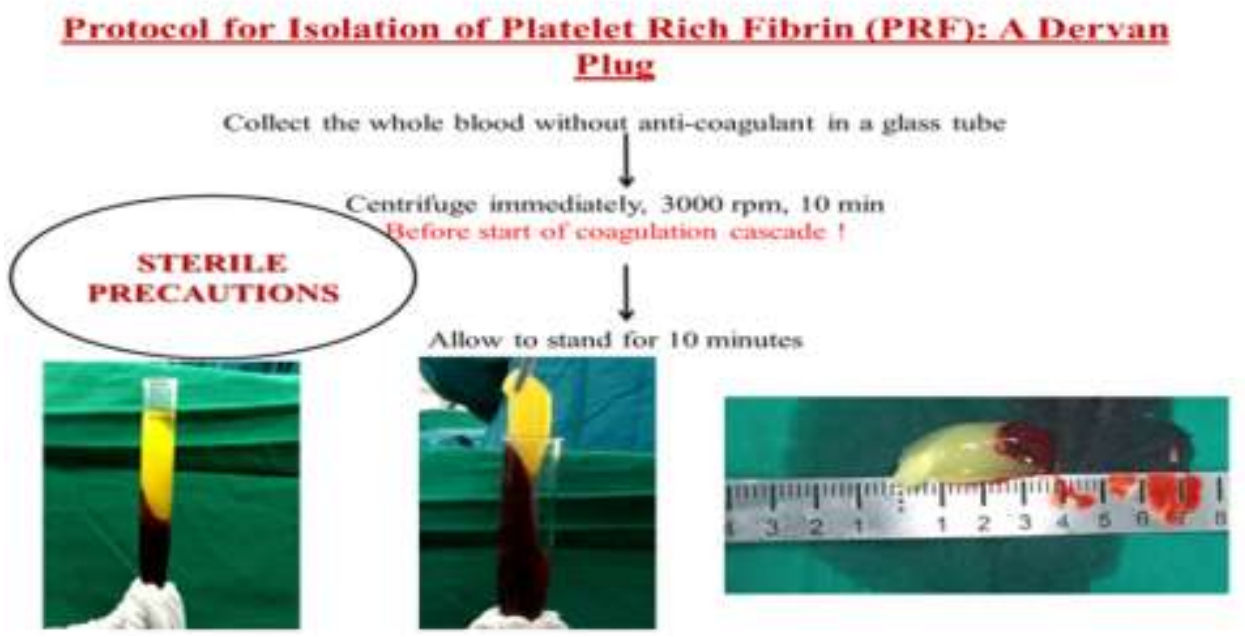

\section{Classification and Evaluation of Platelet Concentrates}

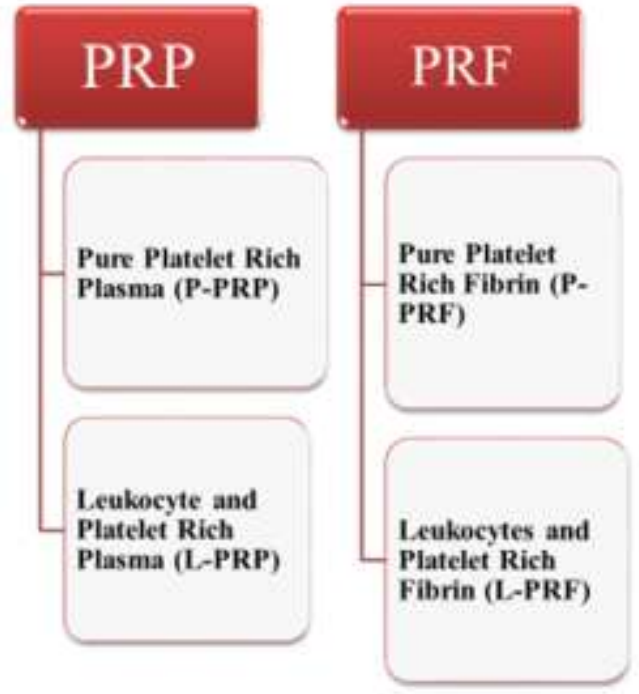

\begin{abstract}
- Platelets plays a key role in regeneration of damaged tissue.

-In 1974 Ross propose that platelet rich plasma (PRP) induces the release of growth factors which stimulates the mitogenic response in bone periosteum during normal healing for repair of the bone.
\end{abstract}

-In 2001 Choukron proposed a platelet concentrate and fibrin in a simpler way without blood modification: Platelet Rich Fibrin (PRF)

The use of Dervan plug as a local wound sealant helped to reduce pre surgical lumbar pain. Improvement in quality of life of these patients was satisfactory. However, in order to establish a correlation between surgical outcomes of surgery and use of Dervan plug of platelet rich fibrin further comparative study with the help of radiological analysis was done. The posterior annular outline was studied in post op MRI and 3 illustrative cases are shown above with excellent results in symptoms and images.

\section{Discussion}

The cost of preparation and expertise and equipment (INR 5 for the test tube \& 5000 for the portable centrifuge alongwith a recurring cost of INR 15 per use of electricity, sterilisation $\&$ use of sterile syringe to extract blood by venepuncture etc.) is minimum and equipment is readily available. It is a safe quick and successful method of obtaining a bioactive plug for healing of the annulus.

We have been able to establish a simple protocol to get a sizable Dervan plug for our use during and at end of surgery. Our ability to remove the causative nuclear fragments trapped in annular fibres and instilling plug at the tear has added to post-operative relief of pain and discomfort and early return to normal mobility. The pre and post-operative images were compared and they showed satisfactory healing of the annulus.

In vitro evidence suggests platelet rich plasma would accelerate local healing. ${ }^{12} 131415$. We have limited experience of injecting plasma inside and around the disc. Platelet rich fibrin plug has been shown to be useful in vitro and vivo to accelerate healing of tissues. It was first introduced by Choukren et al ${ }^{16}$ in dentistry for enhancement of bone matrix formation before implantation etc.in year 2001. It is autogenic and the time 
required for preparation is short (20 minutes by our modified technique). Corso et $\mathrm{al}^{17}$ proposed that PRF acts as bioactive material in dental practices, a blood clot for neovascularisation and promote the tissue architecture.

In vitro study ${ }^{18} 19$ shows PRF supporting bone morphogenic protein releases cytokines and number of potential growth factors (e.g. PDGF-AA, PDGF-BB, TGF- $\beta$, BFGF, VEGF, IGF, bFGF etc.) accelerates the collagen system and promote fast healing of the soft tissue. These released growth factors from PRF could stimulate cell proliferation, fibroblast production, chemotaxis, gene expression and promote the accumulation of ECM, cell migration and angiogenesis.Release cytokines plays key role in the regulation of inflammation phenomenon. From the evidence available from in vitro studies it is expected to orchestrate and accelerate local healing of tissues. ${ }^{2021}$

Short term studies have demonstrated the positive effect on post-operative pain, spasm and discomfort. Further long term studies can evaluate if the in vitro evidence of the stimulant effect of platelets translates into appreciable clinical benefit in terms of healing of the disc and changes in long term outcome after discectomy. The present report is encouraging and should allow us to design more studies with confidence to determine the place of platelet rich fibrin Dervan plug in discogenicback and leg pain.

Platelet rich plasma injections have been tried in patients with chronic back pain with good results. ${ }^{22 .}$ We did not find any reports in the literature of the use of PRF (Platelet rich fibrin) in spine surgery. The present case series is the first report in the literature as far as we are aware of in vivo use of PRF following discectomy and our experience over the last 24 months in inducing healing of the torn annulus and degenerate nucleus pulposus.

We acknowledge help of Ms Bhagyashri Kulkarni MSc in preparation of manuscript.

\section{References}

[1]. Moriguchi Y1, Alimi M1, Khair T1, Manolarakis G1, Berlin C1, Bonassar LJ2, Härtl R1. Biological Treatment Approaches for Degenerative Disk Disease: A Literature Review of In Vivo Animal and Clinical Data. Global Spine J. 2016 Aug;6(5):497-518. doi: 10.1055/s-0036-1571955. Epub 2016 Jan 27.

[2]. Gore S1, Yeung A2 The "inside out" transforaminal technique to treat lumbar spinal pain in an awake and aware patient under local anesthesia: results and a review of the literature. Int J Spine Surg. 2014 Dec 1;8. doi: 10.14444/1028. eCollection 2014.

[3]. Yeung AT1, Gore S2. In-vivo Endoscopic Visualization of Patho-anatomy in Symptomatic Degenerative Conditions of the Lumbar Spine II: Intradiscal, Foraminal, and Central Canal Decompression. Surg Technol Int. 2011 Dec;21:299-319.

[4]. Li P, Jia N, Shen Y, Jin X, Shen Y, Ding W, Zhang W. Experimental study on effect of three different operative ways of annulus fibrosus incision on intervertebral disc biomechanical strength. Zhongguo Xiu Fu Chong Jian Wai Ke Za Zhi. 2016 Feb;30(2):2027.

[5]. Brisby H1. Pathology and possible mechanisms of nervous system response to disc degeneration. J Bone Joint Surg Am. 2006 Apr;88 Suppl 2:68-71.

[6]. Sanchez, E. Anitua, G. Orive, I. Mujika, I. Andia, Platelet-Rich Therapies in the Treatment of Orthopaedic Sport Injuries, Sports Med. 29 (2009) 345-354.

[7]. U. Sheth, N. Simunovic, G. Klein et al., Efficacy of autologous platelet-rich plasma use for orthopaedic indications: a metaanalysis, J Bone Joint Surg Am. 94(4) (2012) 298-307.

[8]. V.Y. Moraes, M. Lenza, M.J. Tamaoki, F. Faloppa, J.C. Bellori, Platelet-rich Therapies for Musculoskeletal Soft Tissue Injuries (Review), Clin Orthop Relat Res. 473(7) (2015) 2207-2213.

[9]. B. Naik, P. Karunakar, M. Jayadev, V.R. Marshal, Role of Platelet rich fibrin in wound healing:A critical review, J Conserv Dent. 16(2013) 284-293.

[10]. S.V. Khiste, R.N. Tari, Platelet-Rich Fibrin as a Biofuel for Tissue Regeneration, ISRM Biomaterials. (2013). http://dx.doi.org/10.5402/2013/627367.

[11]. GORE SYSTEM https://openi.nlm.nih.gov/detailedresult.php?img=PMC4325508 IJSS-8-14444-1028-g015\&req=4

[12]. T. Bielecki, D.M. Dohan Ehrenfest, Leukocyte- and Platelet-Rich Plasma (L-PRP)/Fibrin (L-PRF) in Medicine- Past, Present, Future, Curr Pharm Biotechnol. 13(7) (2012). Editorial

[13]. D.P. Kuffler, Platelet-rich plasma and the elimination of neuropathic pain, Mol Neurobiol. 48(2) (2013) 315-332.

[14]. B.L. Eppley, J.E. Woodell, J. Higging, Platelet quantification and growth factor analysis from platelet-rich plasma: implications for wound healing, Plast Reconstr Surg. 114(6) (2004) 1502-1508.

[15]. G.F. Pierce, T.A. Mustoe, B.M. Altrock, T.F. Deuel, A. Thomason, Role of platelet-derived growth factor in wound healing, J Cell Bichem. 45(4) (1991) 319-326.

[16]. D.M. Dohan, J. Choukroun, A. Diss et al., Platelet-rich fibrin (PRF): A second-generation platelet concentrate. Part IV: Clinical effects on tissue healing, Oral Surg Oral Med Oral Pathol Oral Radiol Endod. 101 (2006) e56-60.

[17]. M.D. Corso, M. Toffler, D.M. Dohan Ehrenfest, Use of Autologous leukocyte and Platelet Rich Fibrin (L-PRF) membrane in PostAvulsion site: - A over View of Choukroun's PRF, JIACD. 1 (2010) 27-38.

[18]. C.Y. Su, Y.P. Kuo, Y.H. Tseng, C.H. Su, T. Burnouf, In vitro release of growth factors from platelet-rich fibrin (PRF): A proposal to optimize the clinical applications of PRF, Oral Surg Oral Med Oral Pathol Oral Radiol Endod. 108 (2009) 56-61.

[19]. S. Roy, J. Driggs, H. Elgharably et al., Platelet-rich fibrin matrix improves wound angiogenesis via inducing endothelial cell proliferation, Wound Repair Regen. 19(2011)753-766.

[20]. J.L. Bron, M.N. Helder, H.J. Meisel, B.J. Van Royen, T.H. Smit, Repair, regenerative and supportive therapies of the annulus fibrosus: achievements and challenges, Eur Spine J. 18 (2009) 301-313.

[21]. G.B. Gullung, J.W. Woodall, M.A. Tucci, J. James, D.A. Black, Platelet-rich plasma effects on degenerative disc disease: analysis of histology and imaging in an animal model, Evid Based Spine Care J. 2(4) (2011) 13-11.

[22]. D. Levi, S. Horn, S. Tyszko, J. Levin, C. Hecht-Leavitt, E. Walko, Intradiscal Platelet-Rich Plasma Injection for Chronic Discogenic Low Back Pain: Preliminary Results from a Prospective Trial, Pain Med. 0 (2015) 1-13. 